EUROPEAN ORGANISATION FOR NUCLEAR RESEARCH

CERN-PPE/94-103

4 July, 1994

\title{
Search for a scalar top quark using the OPAL detector
}

\author{
The OPAL Collaboration
}

\begin{abstract}
A search for a supersymmetric partner of the top quark $\left(\tilde{\mathrm{t}}_{1}\right)$ has been performed by the OPAL experiment in $\mathrm{e}^{+} \mathrm{e}^{-}$collisions at LEP. The integrated luminosity of the data sample analysed was $69.1 \mathrm{pb}^{-1}$, which corresponds to $1.68 \times 10^{6}$ produced $\mathrm{Z}^{0} \rightarrow \mathrm{q} \overline{\mathrm{q}}$ events. No $\tilde{\mathrm{t}}_{1}$ candidates have been found. This study excludes the existence of the $\tilde{t}_{1}$ with a mass below $45.1 \mathrm{GeV}$ at $95 \%$ C.L., where the mixing angle of left- and right-handed partners is smaller than 0.85 rad or greater than $1.15 \mathrm{rad}$, and the mass difference between the $\tilde{t}_{1}$ and the lightest neutralino is greater than $5 \mathrm{GeV}$.
\end{abstract}

(Submitted to Physics Letters B) 


\section{The OPAL Collaboration}

R. Akers ${ }^{16}$, G. Alexander ${ }^{23}$, J. Allison ${ }^{16}$, K.J. Anderson ${ }^{9}$, S. Arcelli ${ }^{2}$, S. Asai ${ }^{24}$, A. Astbury ${ }^{28}$, D. Axen ${ }^{29}$, G. Azuelos ${ }^{18, a}$, A.H. Ball ${ }^{17}$, E. Barberio ${ }^{26}$, R.J. Barlow ${ }^{16}$, R. Bartoldus ${ }^{3}$, J.R. Batley ${ }^{5}$, G. Beaudoin ${ }^{18}$, A. Beck ${ }^{23}$, G.A. Beck ${ }^{13}$, J. Becker ${ }^{10}$, C. Beeston ${ }^{16}$, T. Behnke ${ }^{27}$, K.W. Bell ${ }^{20}$, G. Bella ${ }^{23}$, P. Bentkowski ${ }^{18}$, S. Bentvelsen ${ }^{8}$, P. Berlich ${ }^{10}$, S. Bethke $^{32}$, O. Biebel ${ }^{32}$, I.J. Bloodworth ${ }^{1}$, P. Bock ${ }^{11}$, H.M. Bosch ${ }^{11}$, M. Boutemeur ${ }^{18}$, S. Braibant ${ }^{12}$, P. Bright-Thomas ${ }^{25}$, R.M. Brown ${ }^{20}$, A. Buijs ${ }^{8}$, H.J. Burckhart ${ }^{8}$, C. Burgard ${ }^{27}$,

P. Capiluppi ${ }^{2}$, R.K. Carnegie ${ }^{6}$, A.A. Carter $^{13}$, J.R. Carter ${ }^{5}$, C.Y.Chang ${ }^{17}$, C. Charlesworth ${ }^{6}$, D.G. Charlton ${ }^{8}$, S.L. $\mathrm{Chu}^{4}$, P.E.L. Clarke ${ }^{15}$, J.C. Clayton ${ }^{1}$, S.G. Clowes ${ }^{16}$, I. Cohen ${ }^{23}$, J.E. Conboy ${ }^{15}$, M. Coupland ${ }^{14}$, M. Cuffiani ${ }^{2}$, S. Dado ${ }^{22}$, C. Dallapiccola ${ }^{17}$, G.M. Dallavalle ${ }^{2}$, C. Darling ${ }^{31}$, S. De Jong ${ }^{13}$, H. Deng ${ }^{17}$, M. Dittmar ${ }^{4}$, M.S. Dixit ${ }^{7}$, E. do Couto e Silva ${ }^{12}$, J.E. Duboscq ${ }^{8}$, E. Duchovni ${ }^{26}$, G. Duckeck ${ }^{8}$, I.P. Duerdoth ${ }^{16}$, U.C. Dunwoody ${ }^{5}$, P.A. Elcombe ${ }^{5}$, P.G. Estabrooks ${ }^{6}$, E. Etzion ${ }^{23}$, H.G.Evans ${ }^{9}$, F. Fabbri ${ }^{2}$, B. Fabbro ${ }^{21}$, M. Fanti ${ }^{2}$,

M. Fierro ${ }^{2}$, M. Fincke-Keeler ${ }^{28}$, H.M. Fischer ${ }^{3}$, P. Fischer ${ }^{3}$, R. Folman ${ }^{26}$, D.G. Fong ${ }^{17}$, M. Foucher ${ }^{17}$, H. Fukui ${ }^{24}$, A. Fürtjes ${ }^{8}$, P. Gagnon ${ }^{6}$, A. Gaidot ${ }^{21}$, J.W. Gary ${ }^{4}$, J. Gascon ${ }^{18}$, N.I. Geddes ${ }^{20}$, C. Geich-Gimbel ${ }^{3}$, S.W. Gensler ${ }^{9}$, F.X. Gentit ${ }^{21}$, T. Geralis ${ }^{20}$, G. Giacomelli ${ }^{2}$, P. Giacomelli ${ }^{4}$, R. Giacomelli ${ }^{2}$, V. Gibson ${ }^{5}$, W.R. Gibson ${ }^{13}$, J.D. Gillies ${ }^{20}$, J. Goldberg ${ }^{22}$, D.M. Gingrich ${ }^{30, a}$, M.J. Goodrick ${ }^{5}$, W. Gorn ${ }^{4}$, C. Grandi ${ }^{2}$, P. Grannis ${ }^{8}$, E. Gross ${ }^{26}$, J. Hagemann ${ }^{27}$, G.G.Hanson ${ }^{12}$, M. Hansroul ${ }^{8}$, C.K. Hargrove ${ }^{7}$, J. Hart ${ }^{8}$, P.A. Hart ${ }^{9}$,

M. Hauschild ${ }^{8}$, C.M.Hawkes ${ }^{8}$, E. Heflin ${ }^{4}$, R.J.Hemingway ${ }^{6}$, G. Herten ${ }^{10}$, R.D. Heuer ${ }^{8}$, J.C.Hill ${ }^{5}$, S.J. Hillier ${ }^{8}$, T. Hilse ${ }^{10}$, D.A.Hinshaw ${ }^{18}$, P.R.Hobson ${ }^{25}$, D. Hochman ${ }^{26}$, A. Höcker ${ }^{3}$, R.J. Homer ${ }^{1}$, A.K.Honma ${ }^{28, a}$, R.E. Hughes-Jones ${ }^{16}$,

R. Humbert ${ }^{10}$, P. Igo-Kemenes ${ }^{11}$, H. Ihssen ${ }^{11}$, D.C. Imrie ${ }^{25}$, A. Jawahery ${ }^{17}$, P.W. Jeffreys ${ }^{20}$, H. Jeremie ${ }^{18}$, M. Jimack ${ }^{1}$, M. Jones ${ }^{6}$, R.W.L. Jones ${ }^{8}$, P. Jovanovic ${ }^{1}$, C. Jui ${ }^{4}$, D. Karlen ${ }^{6}$, K. Kawagoe ${ }^{24}$, T. Kawamoto ${ }^{24}$, R.K. Keeler ${ }^{28}$, R.G.Kellogg ${ }^{17}$, B.W.Kennedy ${ }^{20}$, B. King ${ }^{8}$, J. King ${ }^{13}$, S. Kluth ${ }^{5}$, T. Kobayashi ${ }^{24}$,

M. Kobe ${ }^{10}$, D.S. Koetke ${ }^{8}$, T.P. Kokott ${ }^{3}$, S. Komamiya ${ }^{24}$, R. Kowalewski ${ }^{8}$,

R. Howard ${ }^{29}$, P. Krieger ${ }^{6}$, J. von Krogh ${ }^{11}$, P. Kyberd ${ }^{13}$, G.D. Lafferty ${ }^{16}$, H. Lafoux ${ }^{8}$, R. Lahmann ${ }^{17}$, J. Lauber ${ }^{8}$, J.G. Layter ${ }^{4}$, P. Leblanc ${ }^{18}$, P. Le Du ${ }^{21}$, A.M. Lee ${ }^{31}$, E. Lefebvre ${ }^{18}$, M.H. Lehto ${ }^{15}$, D. Lellouch ${ }^{26}$, C. Leroy ${ }^{18}$, J. Letts ${ }^{4}$, L. Levinson ${ }^{26}$, Z. $\operatorname{Li}^{12}$, F. Liu ${ }^{29}$, S.L. Lloyd ${ }^{13}$, F.K. Loebinger ${ }^{16}$, G.D. Long ${ }^{17}$, B. Lorazo ${ }^{18}$, M.J. Losty ${ }^{7}$, X.C. Lou ${ }^{8}$, J. Ludwig ${ }^{10}$, A. Luig ${ }^{10}$, M. Mannelli ${ }^{8}$, S. Marcellini' ${ }^{2}$, C. Markus ${ }^{3}$, A.J. Martin ${ }^{13}$, J.P. Martin ${ }^{18}$, T. Mashimo ${ }^{24}$, P. Mättig ${ }^{3}$, U. Maur ${ }^{3}$, J. McKenna ${ }^{29}$, T.J.McMahon ${ }^{1}$, A.I. McNab ${ }^{13}$, J.R. McNutt ${ }^{25}$, F. Meijers ${ }^{8}$, F.S. Merritt ${ }^{9}$, H. Mes ${ }^{7}$, A. Michelini ${ }^{8}$,

R.P. Middleton ${ }^{20}$, G. Mikenberg ${ }^{26}$, J. Mildenberger ${ }^{6}$, D.J. Miller ${ }^{15}$, R. Mir $^{26}$, W. Mohr ${ }^{10}$, C. Moisan ${ }^{18}$, A. Montanari ${ }^{2}$, T. Mori ${ }^{24}$, M. Morii ${ }^{24}$, U. Müller ${ }^{3}$, B. Nellen ${ }^{3}$, B. Nijjhar ${ }^{16}$, S.W. O'Neale ${ }^{1}$, F.G. Oakham ${ }^{7}$, F. Odorici ${ }^{2}$, H.O. Ogren ${ }^{12}$, C.J. Oram ${ }^{28, a}$, M.J. Oreglia ${ }^{9}$, S. Orito ${ }^{24}$, J.P. Pansart ${ }^{21}$, G.N.Patrick ${ }^{20}$, M.J. Pearce ${ }^{1}$, P. Pfister ${ }^{10}$, P.D. Phillips ${ }^{16}$, J.E. Pilcher ${ }^{9}$, J. Pinfold ${ }^{30}$, D. Pitman ${ }^{28}$, D.E. Plane ${ }^{8}$, P. Poffenberger ${ }^{28}$, B. Poli ${ }^{2}$, A. Posthaus ${ }^{3}$, T.W. Pritchard ${ }^{13}$, H. Przysiezniak ${ }^{18}$, M.W. Redmond ${ }^{8}$, D.L. Rees ${ }^{8}$, D. Rigby ${ }^{1}$, 
M. Rison ${ }^{5}$, S.A. Robins ${ }^{13}$, D. Robinson ${ }^{5}$, J.M. Roney ${ }^{28}$, E. $\operatorname{Ros}^{8}$, S. Rossberg $^{10}$, A.M. Rossi ${ }^{2}$, M. Rosvick ${ }^{28}$, P. Routenburg ${ }^{30}$, Y. Rozen $^{8}$, K. Runge $^{10}$, O. Runolfsson ${ }^{8}$, D.R.Rust ${ }^{12}$, M.Sasaki ${ }^{24}$, C.Sbarra ${ }^{2}$, A.D.Schaile ${ }^{8}$, O.Schaile ${ }^{10}$, F.Scharf ${ }^{3}$, P.Scharff-Hansen ${ }^{8}$, P. Schenk ${ }^{4}$, B.Schmitt ${ }^{3}$, H. von der Schmitt ${ }^{11}$, M. Schröder ${ }^{12}$, H.C.Schultz-Coulon ${ }^{10}$, P.Schütz ${ }^{3}$, M.Schulz ${ }^{8}$, C.Schwick ${ }^{27}$, J.Schwiening ${ }^{3}$, W.G.Scott ${ }^{20}$, M.Settles ${ }^{12}$, T.G.Shears ${ }^{5}$, B.C.Shen ${ }^{4}$, C.H. Shepherd-Themistocleous ${ }^{7}$, P.Sherwood ${ }^{15}$, G.P.Siroli ${ }^{2}$, A.Skillman ${ }^{16}$, A.Skuja ${ }^{17}$, A.M.Smith ${ }^{8}$, T.J.Smith ${ }^{28}$, G.A.Snow ${ }^{17}$, R.Sobie ${ }^{28}$, R.W.Springer ${ }^{17}$, M.Sproston ${ }^{20}$, A.Stahl ${ }^{3}$, C.Stegmann ${ }^{10}$, K.Stephens ${ }^{16}$, J.Steuerer $^{28}$, B.Stockhausen ${ }^{3}$, R.Ströhmer ${ }^{11}$, D.Strom ${ }^{19}$, P.Szymanski ${ }^{20}$, H. Takeda ${ }^{24}$, T. Takeshita ${ }^{24}$, S. Tarem ${ }^{26}$, M. Tecchio ${ }^{9}$, P. Teixeira-Dias ${ }^{11}$, N. Tesch ${ }^{3}$, M.A. Thomson ${ }^{15}$, S. Towers ${ }^{6}$, T. Tsukamoto ${ }^{24}$, M.F. Turner-Watson ${ }^{8}$, D. Van den plas ${ }^{18}$, R. Van Kooten ${ }^{12}$, G. Vasseur ${ }^{21}$, M. Vincter ${ }^{28}$, A. Wagner ${ }^{27}$, D.L. Wagner ${ }^{9}$, C.P. Ward ${ }^{5}$, D.R. Ward ${ }^{5}$, J.J. Ward ${ }^{15}$, P.M. Watkins ${ }^{1}$, A.T. Watson ${ }^{1}$, N.K. Watson ${ }^{7}$, P. Weber ${ }^{6}$, P.S. Wells ${ }^{8}$, N. Wermes ${ }^{3}$, B. Wilkens ${ }^{10}$, G.W.Wilson ${ }^{4}$, J.A.Wilson ${ }^{1}$, V-H. Winterer ${ }^{10}$, T. Wlodek ${ }^{26}$, G. Wolf ${ }^{26}$, S. Wotton ${ }^{11}$, T.R. Wyatt ${ }^{16}$, A. Yeaman ${ }^{13}$, G. Yekutieli ${ }^{26}$, M. Yurko ${ }^{18}$, W. Zeuner ${ }^{8}$, G.T. Zorn ${ }^{17}$.

${ }^{1}$ School of Physics and Space Research, University of Birmingham, Birmingham B15 2TT, UK

${ }^{2}$ Dipartimento di Fisica dell' Università di Bologna and INFN, I-40126 Bologna, Italy

${ }^{3}$ Physikalisches Institut, Universität Bonn, D-53115 Bonn, Germany

${ }^{4}$ Department of Physics, University of California, Riverside CA 92521, USA

${ }^{5}$ Cavendish Laboratory, Cambridge CB3 0HE, UK

${ }^{6}$ Carleton University, Department of Physics, Colonel By Drive, Ottawa, Ontario K1S 5B6, Canada

${ }^{7}$ Centre for Research in Particle Physics, Carleton University, Ottawa, Ontario K1S 5B6, Canada

${ }^{8} \mathrm{CERN}$, European Organisation for Particle Physics, CH-1211 Geneva 23, Switzerland

${ }^{9}$ Enrico Fermi Institute and Department of Physics, University of Chicago, Chicago IL 60637, USA

${ }^{10}$ Fakultät für Physik, Albert Ludwigs Universität, D-79104 Freiburg, Germany ${ }^{11}$ Physikalisches Institut, Universität Heidelberg, D-69120 Heidelberg, Germany ${ }^{12}$ Indiana University, Department of Physics, Swain Hall West 117, Bloomington IN 47405, USA

${ }^{13}$ Queen Mary and Westfield College, University of London, London E1 4NS, UK ${ }^{14}$ Birkbeck College, London WC1E 7HV, UK

${ }^{15}$ University College London, London WC1E 6BT, UK

${ }^{16}$ Department of Physics, Schuster Laboratory, The University, Manchester M13 9PL, UK

${ }^{17}$ Department of Physics, University of Maryland, College Park, MD 20742, USA ${ }^{18}$ Laboratoire de Physique Nucléaire, Université de Montréal, Montréal, Quebec 
H3C 3J7, Canada

${ }^{19}$ University of Oregon, Department of Physics, Eugene OR 97403, USA

${ }^{20}$ Rutherford Appleton Laboratory, Chilton, Didcot, Oxfordshire OX11 0QX, UK

${ }^{21}$ CEA, DAPNIA/SPP, CE-Saclay, F-91191 Gif-sur-Yvette, France

${ }^{22}$ Department of Physics, Technion-Israel Institute of Technology, Haifa 32000, Israel

${ }^{23}$ Department of Physics and Astronomy, Tel Aviv University, Tel Aviv 69978, Israel

${ }^{24}$ International Centre for Elementary Particle Physics and Department of Physics, University of Tokyo, Tokyo 113, and Kobe University, Kobe 657, Japan

${ }^{25}$ Brunel University, Uxbridge, Middlesex UB8 3PH, UK

${ }^{26}$ Particle Physics Department, Weizmann Institute of Science, Rehovot 76100 , Israel

${ }^{27}$ Universität Hamburg/DESY, II Institut für Experimental Physik, Notkestrasse 85, D-22607 Hamburg, Germany

${ }^{28}$ University of Victoria, Department of Physics, P O Box 3055, Victoria BC V8W 3P6, Canada

${ }^{29}$ University of British Columbia, Department of Physics, Vancouver BC V6T 1Z1, Canada

${ }^{30}$ University of Alberta, Department of Physics, Edmonton AB T6G 2J1, Canada

${ }^{31}$ Duke University, Dept of Physics, Durham, NC 27708-0305, USA

${ }^{32}$ Technische Hochschule Aachen, III Physikalisches Institut, Sommerfeldstrasse 26-28, D-52056 Aachen, Germany

aAlso at TRIUMF, Vancouver, Canada V6T $2 \mathrm{~A} 3$

\section{Introduction}

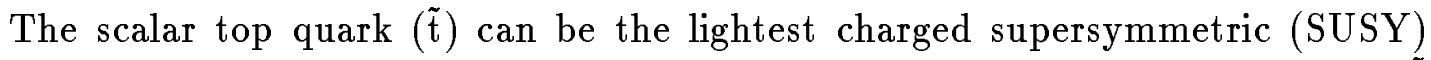
particle for two reasons $[1,2]$. Firstly, one loop radiative corrections to the $\tilde{t}$ mass through Higgsino-quark loops and Higgs-squark loops are always negative. The correction is large for the top quark mass of about $160 \mathrm{GeV}$, as predicted from the precise measurements of $\mathrm{Z}^{0}$ parameters at LEP [3], and supported by the recent direct measurement reported by the CDF collaboration [4]. Secondly, the supersymmetric partners of the right-handed and left-handed top quarks $\left(\tilde{t}_{L}\right.$ and $\left.\tilde{t}_{R}\right)$ mix, and the resultant two mass eigenstates $\left(\tilde{t}_{1}\right.$ and $\left.\tilde{t}_{2}\right)$ have a large mass splitting. The lighter mass eigenstate $\left(\tilde{\mathrm{t}}_{1}\right)$ can be lighter than any other charged SUSY particle, and lighter than the top quark itself $[1,2]$. The $\tilde{t}_{1}$ mass can be close to that of the lightest SUSY particle (the neutralino or $\tilde{\chi}_{1}^{0}$ ) for some regions of the allowed parameter space [5]. We assume in this paper that the lightest SUSY particle carries no electric charge and does not interact strongly.

Lower limits on scalar quark masses from $\mathrm{p} \overline{\mathrm{p}}$ colliders [6] were obtained with the assumptions that all the scalar quark masses of the five or six flavours are degenerate, and that masses of the left and right-handed partners were equal. Such 
assumptions are invalid for the $\tilde{t}_{1}$. Furthermore, if the mass difference between $\tilde{\mathrm{t}}_{1}$ and $\tilde{\chi}_{1}^{0}$ is below about $40 \mathrm{GeV}$, missing transverse energy $\mathrm{E}_{T}$ and transverse energy $E_{T}$ of jets coming from $\tilde{t}_{1}$ decays are too small to be distinguished from the background at $\mathrm{p} \overline{\mathrm{p}}$ colliders. Searches at $\mathrm{e}^{+} \mathrm{e}^{-}$colliders are sensitive for small $\tilde{\mathrm{t}}_{1}-\tilde{\chi}_{1}^{0}$ mass differences. Scalar quark searches have been made by DELPHI and MARK II [7] at the $Z^{0}$ resonance, with the assumption that masses of left-handed and right-handed partners of a quark are equal. This assumption is not valid for the $\tilde{t}_{1}$ search. Limits generally valid for the $\tilde{t}_{1}$ were obtained at lower centre of mass energies, at PETRA and TRISTAN $[8,9,10]$.

In this paper, we report on a direct search for $\tilde{t}_{1}$ using the OPAL detector, for the full range of the possible $Z^{0} \tilde{\mathrm{t}}_{1} \tilde{\tilde{t}}_{1}$ coupling strength. As described in more detail below, the $\tilde{t}_{1}$ and $\tilde{t}_{1}$ decay products contain $\tilde{\chi}_{1}^{0}$ 's which carry away a large fraction of the energy and momentum. As the $\tilde{\chi}_{1}^{0}$ is unobservable, the $\tilde{\mathrm{t}}_{1} \tilde{\tilde{t}}_{1}$ events would be characterized by two jets which are not back-to-back, and by relatively small visible energy.

\section{Phenomenology and Simulation of the $\tilde{\mathbf{t}}_{1}$ Events}

\subsection{Production and Decay of the $\tilde{\mathbf{t}}_{1}$}

Scalar top pairs could be produced in $\mathrm{e}^{+} \mathrm{e}^{-}$annihilation via a $\mathrm{Z}^{0}$ boson or a virtual photon. The total cross section including both the first order QCD and QED corrections [1] has been calculated as a function of the scalar top mass $m_{\tilde{\mathrm{t}}_{1}}$ and the mixing angle $\theta_{\text {mix }}$, where $\theta_{\text {mix }}$ is defined by $\tilde{\mathrm{t}}_{1}=\tilde{\mathrm{t}}_{\mathrm{L}} \cos \theta_{\text {mix }}+\tilde{\mathrm{t}}_{\mathrm{R}} \sin \theta_{\text {mix }}$. The coupling between the $\tilde{t}_{1}$ and the $Z^{0}$ boson depends on the mixing angle which is determined by the top quark mass and the soft SUSY breaking parameters. For $\theta_{\text {mix }}$ close to $0.98\left(\cos ^{2} \theta_{\text {mix }}=\frac{4}{3} \sin ^{2} \overline{\theta_{\mathrm{W}}}\right), \tilde{\mathrm{t}}_{1}$ decouples from the $\mathrm{Z}^{0}$ boson, and $\tilde{\mathrm{t}}_{1} \overline{\tilde{t}}_{1}$ can be produced only via a virtual $\gamma(\sigma \sim \mathcal{O}(1) \mathrm{pb})$. Otherwise, a large cross section of $\mathcal{O}(100) \mathrm{pb}$ is expected for $\theta_{\text {mix }}$ close to $\frac{1}{4} \pi$ (full mixing state). In this analysis the masses of the $\tilde{t}_{1}$ and the $\tilde{\chi}_{1}^{0}$, and the mixing angle are treated as free parameters. As the spinless $\tilde{t}_{1}$ is pair produced through a $\mathrm{Z}^{0}$ boson or a virtual photon, the angular distribution $d \sigma / d \cos \theta$ is proportional to $\sin ^{2} \theta$, where $\theta$ is the polar angle between the $\tilde{t}_{1}$ momentum direction and the beam axis.

The $\tilde{t}_{1}$ decays into the lightest SUSY particle $\left(\tilde{\chi}_{1}^{0}\right)$ and non-SUSY particles. The flavour changing two body decay $\tilde{t}_{1} \rightarrow c \tilde{\chi}_{1}^{0}$ would occur via one-loop processes. The decay width of this mode is estimated to be [1]

$$
\Gamma\left(\tilde{\mathrm{t}}_{1} \rightarrow c \tilde{\chi}_{1}^{0}\right)=(0.3 \sim 3) \times 10^{-10} m_{\tilde{\mathrm{t}}_{1}}\left(1-\frac{m_{\tilde{\chi}_{1}^{0}}{ }^{2}}{m_{\tilde{\mathrm{t}}_{1}}{ }^{2}}\right)^{2}
$$

Three-body decays of the $\tilde{\mathrm{t}}_{1}$ containing scalar leptons $\left(\tilde{\mathrm{t}}_{1} \rightarrow \tilde{\nu} \ell^{+} b, \tilde{\mathrm{t}}_{1} \rightarrow \tilde{\ell}^{+} \nu b\right)$ are not considered here, since a light $\tilde{\ell}\left(m_{\tilde{\ell}} \lesssim 45 \mathrm{GeV}\right)$ and a light $\tilde{\nu}\left(m_{\tilde{\nu}} \lesssim 40 \mathrm{GeV}\right)$ have been already excluded [11]. 
The four-body decay $\tilde{\mathrm{t}}_{1} \rightarrow b \tilde{\chi}_{1}^{0} f_{1} \bar{f}_{2}$ would occur either through the process of $\tilde{\mathrm{t}}_{1} \rightarrow b \tilde{\chi}_{1}^{+} \rightarrow b \mathrm{~W}^{+} \tilde{\chi}_{1}^{0}$ or $\tilde{\mathrm{t}}_{1} \rightarrow t \tilde{\chi}_{1}^{0} \rightarrow b \mathrm{~W}^{+} \tilde{\chi}_{1}^{0}$. The decay width is estimated to be

$$
\Gamma\left(\tilde{\mathrm{t}}_{1} \rightarrow b \tilde{\chi}_{1}^{0} f_{1} \bar{f}_{2}\right) \sim \mathcal{O}\left(10^{-14}\right) m_{\tilde{\mathrm{t}}_{1}}
$$

for $\tilde{\chi}_{1}^{ \pm}$mass of about $50 \mathrm{GeV}$ which is close to the current lower mass limit of $47 \mathrm{GeV}$ given by the LEP experiments [11]. The decay width of the process via a virtual top quark is further suppressed due to the large top quark mass.

Thus the dominant decay mode of the $\tilde{t}_{1}$ would be the flavour changing mode, $\tilde{\mathrm{t}}_{1} \rightarrow c \tilde{\chi}_{1}^{0}$, and this remains true in the region near the kinematical boundary of $m_{\tilde{\mathrm{t}}_{1}}=m_{c}+m_{\tilde{\chi}_{1}^{0}}$, where the four body decay modes containing $b$-quark and $\tilde{\chi}_{1}^{0}$ in the final state are kinematically forbidden because $m_{b}>m_{c}$. Therefore, only the $\tilde{\mathrm{t}}_{1} \rightarrow c \tilde{\chi}_{1}^{0}$ mode is considered in this paper. Because the lifetime of the $\tilde{\mathrm{t}}_{1}$ is much longer than the typical time scale of the hadronisation, the $\tilde{t}_{1}$ would hadronise to form a $\tilde{\mathrm{t}}_{1}$-hadron before the $\tilde{\mathrm{t}}_{1}$ decays.

The width of the $\tilde{t}_{1} \overline{\tilde{t}}_{1}$ bound state has been calculated to be approximately 80 $\mathrm{keV}[12,13]$. The threshold for the open $\tilde{\mathrm{t}}_{1}$-hadron production is about $0.6 \mathrm{GeV}$ higher than $2 m_{\tilde{\mathrm{t}}_{1}}$ [12]. The narrowness of the bound state prevents it from having any observable effects in the open $\tilde{t}_{1}$-hadron production.

\subsection{Event Simulation}

The Monte Carlo simulation of the production and the decay of $\tilde{t}_{1}$ was performed as follows. Events with a $\tilde{\mathrm{t}}_{1} \overline{\tilde{t}}_{1}$ pair were generated taking into account initial state radiation [14]. The hadronisation process is subsequently performed to produce colourless $\tilde{\mathrm{t}}_{1}$-hadrons and other fragment products according to the Lund string fragmentation scheme (JETSET 7.3) [14, 15]. Parameters of the perturbative QCD and fragmentation processes in the model were optimised using event shape distributions of hadronic $Z^{0}$ decays measured using OPAL data [16]. The fragmentation of the $\tilde{t}_{1}$ was performed using the fragmentation function proposed by Peterson et al. $[14,17]$, where the parameter $\epsilon_{\tilde{\mathrm{t}}_{1}}$ is set to be

$$
\epsilon_{\tilde{\mathrm{t}}_{1}}=\epsilon_{\mathrm{b}} \frac{m_{\mathrm{b}}^{2}}{m_{\tilde{\mathrm{t}}_{1}}^{2}}, \quad\left(\epsilon_{\mathrm{b}}=0.0057, m_{\mathrm{b}}=5 \mathrm{GeV}\right) .
$$

The $\tilde{t}_{1}$-hadron is composed of a $\tilde{t}_{1}$ and a spectator quark or a diquark. The $\tilde{t}_{1}$ decays to a charm quark and a $\tilde{\chi}_{1}^{0}$. A colour force string was stretched between the charm quark and the spectator. This colour singlet system was hadronised in the manner of the Lund scheme $[14,15]$. Gluon bremsstrahlung (QCD parton showering) was allowed in this process. The generated events were processed by the full detector simulation program and were reconstructed with the same program as the data. 


\section{The OPAL Detector}

The OPAL detector, which is described in detail in [19], is a solenoidal detector with a pressurised central tracking system operating in a $0.435 \mathrm{~T}$ magnetic field. A lead-glass electromagnetic calorimeter together with presamplers and time-offlight scintillators is located outside the magnet coil and pressure vessel. The magnet return yoke is instrumented for hadron calorimetry and is surrounded by external muon chambers. Calorimeters close to the beam axis measure luminosity and complete the acceptance.

A right-handed coordinate system is adopted, where the $x$-axis points to the center of the LEP ring, and positive $z$ is along the electron beam direction. The angles $\theta$ and $\phi$ are the polar and azimuthal angles, respectively. The detector features of relevance to this analysis are described briefly below.

The central tracking system consists of a vertex drift chamber, a jet chamber, $z$-chambers and a silicon micro-vertex detector. In the range $|\cos \theta|<0.73,159$ points are measured in the jet chamber along each track and at least 20 points on a track are obtained over $96 \%$ of the full solid angle.

The forward detectors (FD) are lead-scintillator sandwich calorimeters 24 radiation lengths deep. They are located on both sides of the interaction point and cover the polar angle region between 39 and $150 \mathrm{mrad}$. In 1993 new silicon tungsten calorimeters ( $\mathrm{SW}$ ) are installed in the polar angle region between 26 and 59 mrad.

The electromagnetic calorimeter (EM) consists of a cylindrical array of 9440 lead-glass blocks of 24.6 radiation lengths thickness (for the barrel), and 2264 lead-glass blocks of 20 radiation lengths thickness (for the end-cap). With excellent hermeticity, these detectors cover the full azimuthal angular range in the polar angle range of $|\cos \theta|<0.82$ for the barrel and $0.81<|\cos \theta|<0.984$ for the endcaps. The barrel lead-glass blocks have a pointing geometry. To achieve good hermeticity, the small $1 \mathrm{~mm}$ gaps between the lead-glass blocks do not point exactly to the interaction point. The hermeticity of the endcap calorimeter is due to its non-pointing geometry.

The hadron calorimeter is made up of three sections: the barrel, the endcap and the pole tip, which together cover the region $|\cos \theta|<0.99$. The magnet return yoke is instrumented with limited streamer tubes in the barrel and endcap sections, and with thin, high gain chambers in the pole tips. There are nine layers of chambers in the barrel, eight in the endcap, and ten in the pole tips. These are read out with narrow strips, and by pads which are grouped together to form towers. The strips are used for tracking, and for muon identification. The towers are used for energy measurement, for which the resolution is $\sigma / E=120 \% / \sqrt{E}$ $(E$ in $\mathrm{GeV})$.

There are at least seven, and in most regions eight, absorption lengths of 
material between the interaction point and the muon detectors. Muons with momenta above $3 \mathrm{GeV}$, usually penetrate to the muon chambers. The muon barrel detector covers the region $|\cos \theta|<0.7$. It is composed of four layers of planar drift chambers, with cylindrical geometry. The muon endcap detector covers the polar angle range $0.67<|\cos \theta|<0.98$. It is composed of two planes of limited streamer tube arrays at each end of the detector. The two muon detector subsystems cover $93 \%$ of the full solid angle.

\section{Event Selection}

The present analysis is based on the data which were taken during the period of 1991-1993 at centre of mass energies around the $\mathrm{Z}^{0}$ peak. The integrated luminosity of the data sample collected was $69.1 \mathrm{pb}^{-1}$, which corresponds to $1.68 \times 10^{6}$ produced $Z^{0} \rightarrow q \bar{q}$ events.

When calculating the event variables such as thrust axis polar angle $\theta_{\text {thrust }}$, visible energy $E_{\text {vis }}$ etc., the following charged track selection and calorimeter cluster selection were applied.

The quality requirements of charged tracks used in this analysis are the same as in the $\mathrm{Z}^{0}$ line shape analysis [20]. It was further required that the track contains more than $50 \%$ of the possible hits for a track at the given polar angle, if the momentum of the charged track is greater than $1 \mathrm{GeV}$, and if no cluster in the electromagnetic calorimeter is associated to the track.

The selection of electromagnetic clusters in the EM is given elsewhere [20]. The energy threshold is set to $0.1 \mathrm{GeV}$ for the barrel and $0.2 \mathrm{GeV}$ for the endcaps. In the case where an EM cluster has an associated charged track, the cluster energy exceeding the momentum of the associated charged track is used to avoid double counting. If the energy of an EM cluster is smaller than the momentum of the associated charged track, the cluster is not used.

The hadron calorimeters are important to capture the energy carried by $\mathrm{K}_{L}^{0}$ mesons and neutrons. A large momentum-inbalance is occasionally caused by the fluctuation in the energy measurement of clusters in the hadron calorimeter because of the limited energy resolution. Therefore the hadron calorimeters are used only when the missing transverse momentum $P_{t}$ normalised by the visible energy $E_{\text {vis }}$ satisfies the following condition:

$$
\frac{P_{t}(\text { without } \mathrm{HC})}{E_{\text {vis }}(\text { without } \mathrm{HC})}>\frac{P_{t}(\text { with } \mathrm{HC})}{E_{\text {vis }}(\text { with } \mathrm{HC})} \text {. }
$$

Although this criterion biases the $\tilde{\mathrm{t}}_{1} \overline{\tilde{t}}_{1}$ event topology towards smaller $P_{t}$ and hence the selection efficiency is slightly reduced, the high background rejection power of this procedure makes its inclusion in this analysis essential. 
The cuts used to reduce the various types of background events (events from two photon processes, $\mathrm{Z}^{0}$ decays to hadrons and $\tau^{+} \tau^{-}$pairs, four-fermion events and beam-gas interaction events), are described below. The number of events remaining after each selection criterion is listed in Table 1 for data and typical simulated $\tilde{\mathrm{t}}_{1} \overline{\mathrm{t}}_{1}$ events. Table 2 shows the events remaining after successive cuts for the simulated background processes.

1. In order to reject events from two photon processes and events with hard initial state radiation, scattered electrons or positrons as well as initial state photons with transverse momentum of a few $\mathrm{GeV}$ are tagged by using FD and SW detectors. The threshold of the cluster energy of FD and SW is set to $1 \mathrm{GeV}$. Events are rejected if the sum of cluster energies in FD and SW exceeds $2 \mathrm{GeV}$.

2. The polar angle of the thrust axis of the event was required to be in the region $\left|\cos \theta_{\text {thrust }}\right|<0.7$. This reduces background events from two photon processes and hadronic $\mathrm{Z}^{0}$ decays.

3. Events were rejected if all the charged tracks of the event fell within the two back-to-back cones of half-angle $35^{\circ}$ around the thrust axis, and if one of the cones contains only one charged track and the other one contains three or more tracks. This cut reduces $\mathrm{Z}^{0}$ decays into a $\tau^{+} \tau^{-}$pair.

4. The number of jets found was required to be two. The LUCLUS algorithm [14], with jet resolution parameter $d_{\text {join }}$ set to be $\left(1.5+2.0 \times E_{\text {vis }} / \sqrt{s}\right) \mathrm{GeV}$, was used for the jet finding. The $E_{\text {vis }}$-dependent $d_{\text {join }}$ is needed for good jet reconstruction over a wide range of $m_{\tilde{\mathrm{t}}_{1}}$.

5. Both reconstructed jets must have at least two charged particles. This requirement reduces the $\tau^{+} \tau^{-}$background where either $\tau$ decays to one charged particle.

6. Events are rejected if the charged multiplicity of one reconstructed jet is between two and six, and the other jet contains only two oppositely charged tracks identified as electrons or muons. This cut removed four-fermion events of the type $\tau^{+} \tau^{-} \ell^{+} \ell^{-}(\ell=$ e or $\mu)$.

In order to maintain a high search efficiency over a wide range of $m_{\tilde{t}_{1}}$ and $m_{\tilde{\chi}_{1}^{0}}$, the $E_{\text {vis }},\left|\cos \theta_{\text {jet }}\right|$, and $\theta_{\text {Acop }}$ cuts were optimised separately for light $\left(m_{\tilde{\mathrm{t}}_{1}} \lesssim 35\right.$ $\mathrm{GeV})$ and heavy $\left(m_{\tilde{\mathrm{t}}_{1}} \gtrsim 25 \mathrm{GeV}\right) \tilde{\mathrm{t}}_{1}$ searches, where $\theta_{\text {jet }}$ is the polar angle of the jet closer to the beam axis, and $\theta_{\text {Acop }}$ is the complement of the angle between the two jets projected onto the plane perpendicular to the beam axis.

7. Events were accepted if they fell into the following regions in the $E_{\mathrm{vis}}-\theta_{\text {Acop }}$ plane:

- $0.15 \leq E_{\text {vis }} / \sqrt{s} \leq 0.5$

for the light $\tilde{t}_{1}$ search, 
- $0.15-\theta_{\text {Acop }}\left({ }^{\circ}\right) / 2000 \leq E_{\text {vis }} / \sqrt{s} \quad$ for the heavy $\tilde{\mathrm{t}}_{1}$ search.

8. Events are accepted if they fell into the following regions of the $\left|\cos \theta_{\text {jet }}\right|-\theta_{\text {Acop }}$ plane:

- $\left|\cos \theta_{\text {jet }}\right|<0.75$ and

$\theta_{\text {Acop }} \geq 15^{\circ}+20^{\circ} \times\left|\cos \theta_{\text {jet }}\right| \quad$ for the light $\tilde{t}_{1}$ search,

- $\left|\cos \theta_{\text {jet }}\right|<0.75$ and

$\theta_{\text {Acop }} \geq 30^{\circ}+60^{\circ} \times\left|\cos \theta_{\text {jet }}\right| \quad$ for the heavy $\tilde{t}_{1}$ search.

Figures 1 and 2 show the last two cuts for the light and heavy $\tilde{t}_{1}$ searches, respectively. The figures show scatter plots of $E_{\mathrm{vis}}-\theta_{\text {Acop }}$ and $\left|\cos \theta_{\text {jet }}\right|-\theta_{\text {Acop }}$ for data, simulated $\tilde{\mathrm{t}}_{1} \overline{\tilde{\mathrm{t}}}_{1}$ events and for simulated background events (hadronic $\mathrm{Z}^{0}$ decays $[14], \tau^{+} \tau^{-}[21]$, and two photon processes [22]).

For heavy $\tilde{t}_{1}$, the wider possible mass differences of $\tilde{t}_{1}$ and $\tilde{\chi}_{1}^{0}$ give rise to a wider range of $E_{\text {vis }}$ and a large average acoplanarity angle. The $\theta_{\text {Acop }}$ dependent $E_{\text {vis }}$ cut for heavy $\tilde{\mathrm{t}}_{1}$ efficiently reduces the low $E_{\text {vis }}$ background from two photon processes, while maintaining the good detection efficiency for heavy $\tilde{t}_{1}$ and small $\Delta m\left(\equiv m_{\tilde{\mathrm{t}}_{1}}-m_{\tilde{\chi}_{1}^{0}}\right)$. As the mass of the $\tilde{\mathrm{t}}_{1}$ decreases, the range of the $\tilde{\chi}_{1}^{0}$ transverse momentum from the $\tilde{t}_{1}$ flight direction decreases and the average acoplanarity angle decreases, and the range of the visible energy decreases. Furthermore, when $m_{\tilde{\mathrm{t}}_{1}}$ decreases, the variation of the visible energy range with the mass difference becomes even narrower because a large fraction of visible energy is carried by the particles from the hadronisation process of $\tilde{t}_{1}$.

A more rigorous cut on $\theta_{\text {Acop }}$ was made in the heavy $\tilde{t}_{1}$ search to reduce the number of events from two photon processes which were not eliminated by the mild $E_{\text {vis }}$ cut. Although there are no events in the region of large $\theta_{\text {Acop }}$ and large $\left|\cos \theta_{\text {jet }}\right|$ for light $\tilde{\mathrm{t}}_{1}$ search, the $\left|\cos \theta_{\text {jet }}\right|<0.75$ cut is maintained due to the proximity of the events from two photon processes to the lower visible energy cut, with high measured acoplanarity angle. The high value of the acoplanarity angle is a mismeasurement due to the loss of particles down to the beam pipe for those events with large $\left|\cos \theta_{\text {jet }}\right|$.

After all the selection cuts have been applied, no events remain in the real data or simulated background data samples. The expected numbers of events from background sources calculated by extrapolating the distributions of simulated background events into the search region, were estimated to be less than 0.4 for the searches in both mass regions. 


\begin{tabular}{|c|c|c|c|c|}
\hline & \multicolumn{2}{|c|}{ data } & \multicolumn{2}{|c|}{$\tilde{\mathrm{t}}_{1}$ Monte Carlo } \\
\hline & & & $\begin{array}{l}m_{\tilde{\mathrm{t}}_{1}}=20 \mathrm{GeV} \\
m_{\tilde{\chi}_{1}^{0}}=10 \mathrm{GeV}\end{array}$ & $\begin{aligned} m_{\tilde{\mathrm{t}}_{1}} & =42 \mathrm{GeV} \\
m_{\tilde{\chi}_{1}^{0}} & =32 \mathrm{GeV}\end{aligned}$ \\
\hline selection & light & heavy & light & heavy \\
\hline raw data & \multicolumn{2}{|c|}{$6.61 \times 10^{6}$} & 2000 & 2000 \\
\hline (1) FD,SW Veto & \multicolumn{2}{|c|}{$4.02 \times 10^{6}$} & 1990 & 1986 \\
\hline (2) $\left|\cos \theta_{\text {thrust }}\right|<0.7$ & \multicolumn{2}{|c|}{$1.51 \times 10^{6}$} & 1714 & 1451 \\
\hline (3) $1-N$ prong Veto & \multicolumn{2}{|c|}{$1.50 \times 10^{6}$} & 1689 & 1421 \\
\hline (4) $N_{\mathrm{j} e t}=2$ & \multicolumn{2}{|c|}{$4.30 \times 10^{5}$} & 1090 & 1318 \\
\hline (5) Multiplicity cut & \multicolumn{2}{|c|}{$2.70 \times 10^{5}$} & 1005 & 1121 \\
\hline (6) 4-fermion veto & \multicolumn{2}{|c|}{$2.69 \times 10^{5}$} & 965 & 1050 \\
\hline (7) $E_{\text {vis }} \mathrm{cut}$ & 1090 & 261865 & 759 & 1027 \\
\hline (8) $\left|\cos \theta_{\text {jet }}\right|-\theta_{\text {Acop }} \mathrm{cut}$ & 0 & 0 & 356 & 450 \\
\hline
\end{tabular}

Table 1: Event selections for the real data and for the typical $\tilde{t}_{1}$ Monte Carlo events. The raw data includes a large number of single track events from $\mathrm{e}^{+} \mathrm{e}^{-} \rightarrow \mathrm{e}^{+} \mathrm{e}^{-} \mathrm{e}^{+} \mathrm{e}^{-}$and events due to interaction of beam and residual gas in the beam pipe.

\begin{tabular}{|c|c|c|c|c|c|c|}
\hline & \multicolumn{2}{|c|}{$\mathrm{Z}^{0} \rightarrow \mathrm{q} \overline{\mathrm{q}}$} & \multicolumn{2}{|c|}{$\mathrm{Z}^{0} \rightarrow \tau^{+} \tau^{-}$} & \multicolumn{2}{|c|}{$\gamma \gamma$ collision } \\
\hline selection & light & heavy & light & heavy & light & heavy \\
\hline raw data & \multicolumn{2}{|c|}{$1.68 \times 10^{6}$} & \multicolumn{2}{|c|}{$1.00 \times 10^{5}$} & \multicolumn{2}{|c|}{$5.63 \times 10^{5}$} \\
\hline (1) FD,SW Veto & \multicolumn{2}{|c|}{$1.60 \times 10^{6}$} & \multicolumn{2}{|c|}{$1.00 \times 10^{5}$} & \multicolumn{2}{|c|}{$2.75 \times 10^{5}$} \\
\hline (2) $\left|\cos \theta_{\text {thrust }}\right|<0.7$ & \multicolumn{2}{|c|}{$8.17 \times 10^{5}$} & \multicolumn{2}{|c|}{$2.95 \times 10^{4}$} & \multicolumn{2}{|c|}{$6.74 \times 10^{4}$} \\
\hline (3) $1-N$ prong Veto & \multicolumn{2}{|c|}{$8.17 \times 10^{5}$} & \multicolumn{2}{|c|}{$2.07 \times 10^{4}$} & \multicolumn{2}{|c|}{$6.73 \times 10^{4}$} \\
\hline (4) $N_{\mathrm{j} e t}=2$ & \multicolumn{2}{|c|}{$2.75 \times 10^{5}$} & \multicolumn{2}{|c|}{$1.86 \times 10^{4}$} & \multicolumn{2}{|c|}{9377} \\
\hline (5) Multiplicity cut & \multicolumn{2}{|c|}{$2.75 \times 10^{5}$} & \multicolumn{2}{|c|}{1632} & \multicolumn{2}{|c|}{5320} \\
\hline (6) 4-fermion veto & \multicolumn{2}{|c|}{$2.73 \times 10^{5}$} & \multicolumn{2}{|c|}{1357} & \multicolumn{2}{|c|}{4690} \\
\hline (7) $E_{\text {vis }} \mathrm{cut}$ & 822 & $2.73 \times 10^{5}$ & 184 & 1357 & 218 & 242 \\
\hline (8) $\left|\cos \theta_{\text {jet }}\right|-\theta_{\text {Acop }} \operatorname{cut}$ & 0 & 0 & 0 & 0 & 0 & 0 \\
\hline
\end{tabular}

Table 2: Number of events expected after each cut for simulated events of hadronic $\mathrm{Z}^{0}$ decay, $\mathrm{Z}^{0} \rightarrow \tau^{+} \tau^{-}$and for two photon processes normalized to the same luminosity as the data. The large excess of the events in the data before the multiplicity cut (Table 1 ) is due to events from soft two photon processes like $\mathrm{e}^{+} \mathrm{e}^{-} \rightarrow \mathrm{e}^{+} \mathrm{e}^{-} \mathrm{e}^{+} \mathrm{e}^{-}$which is not simulated, but the events are eliminated by the multiplicity cut.

\section{Systematic Errors and Mass Limits}

\subsection{Detection Efficiency}

The detection efficiency $\eta$ depends on $m_{\tilde{\mathrm{t}}_{1}}$ and $m_{\tilde{\chi}_{1}^{0}}$. We generated $\tilde{\mathrm{t}}_{1} \overline{\tilde{\mathrm{t}}}_{1}$ events with full detector simulation for forty-one combinations of $m_{\tilde{\mathrm{t}}_{1}}$ and $m_{\tilde{\chi}_{1}^{0}}$ with emphasis 
on the region where the mass difference is small. The efficiency for arbitrary values of $m_{\tilde{\mathrm{t}}_{1}}$ and $m_{\tilde{\chi}_{1}^{0}}$ is estimated by interpolation. The typical efficiency is $20 \%$ in the mass region $m_{\tilde{\mathrm{t}}_{1}} \geq 25 \mathrm{GeV}$ and $m_{\tilde{\mathrm{t}}_{1}}-m_{\tilde{\chi}_{1}^{0}} \geq 5 \mathrm{GeV}$.

\subsection{Systematic Errors}

The sources of systematic error on the detection efficiency $\eta$ are summarised as follows, where the values of relative errors $(|\Delta \eta| / \eta)$ are given:

1. Statistical error of the $\tilde{t}_{1}$ Monte Carlo Simulation.

2. Fragmentation function for $\tilde{\mathrm{t}}_{1}$ : The multiplicity and the visible energy of the $\tilde{\mathrm{t}}_{1} \overline{\tilde{t}}_{1}$ events depend on the fragmentation function of the $\tilde{\mathrm{t}}_{1}$. The fragmentation scheme by Peterson et al. was used [17], with the fragmentation parameter $\epsilon_{\tilde{\mathrm{t}}_{1}}$ determined by formula $(3)$. The error in $\epsilon_{\tilde{\mathrm{t}}_{1}}$ is propagated from $\frac{\delta \epsilon_{b}}{\epsilon_{b}}={ }_{-0.50}^{+0.70}[24]$ and $\frac{\delta m_{b}}{m_{b}}= \pm 0.06 . \quad \tilde{\mathrm{t}}_{1} \overline{\tilde{t}}_{1}$ pair events were generated and simulated with masses of 10,20 and $30 \mathrm{GeV}$ with $\epsilon_{\tilde{\mathrm{t}}_{1}}$ varied by one standard deviation. The change in the detection efficiency was found to be $13 \%, 10 \%$ and $5 \%$, respectively, for the three masses. The deviation $\left(\frac{|\Delta \eta|}{\eta}\right)$ depends mainly on $m_{\tilde{\mathrm{t}}_{1}}$. The systematic error due to $\epsilon_{\tilde{\mathrm{t}}_{1}}$ for arbitrary $m_{\tilde{\mathrm{t}}_{1}}$ was estimated by interpolating the above values.

The fragmentation function proposed by Bowler [23] was also used, because the shape of the fragmentation function is very different from that of Peterson et al.. The difference in detection efficiency for events using the Bowler fragmentation function and those using the Peterson function was found to be typically $4 \%$. This difference was taken to be the uncertainty due to the parametrization of the $\tilde{t}_{1}$ fragmentation function.

3. Fragmentation of charm quark: The systematic error in the efficiency from the uncertainty in the fragmentation parameter of the charm quark $\left(\epsilon_{c}\right)$ is estimated to be typically $4 \%$ for all combinations of $m_{\tilde{\mathrm{t}}_{1}}$ and $m_{\tilde{\chi}_{1}^{0}}$ by varying $\epsilon_{c}$ within the range $\frac{\delta \epsilon_{c}}{\epsilon_{c}}= \pm 0.4[24]$.

4. Energy scale of calorimeters: The visible energy cut depends on the absolute energy scale of the calorimeters. The efficiencies for the searches were determined after shifting the electromagnetic calorimeter energy scale by $\pm 1 \%$, and the hadron calorimeter energy by $\pm 1.2 / \sqrt{E(\mathrm{GeV})}$. The relative change in efficiency was $3 \%$ for light $\tilde{t}_{1}$ search, and $2 \%$ for the heavy $\tilde{t}_{1}$ search.

5. The systematic error due to the luminosity measurement and the trigger efficiencies are negligible.

In addition to the systematic errors in the efficiency, the error of the theoretical calculation of the $\tilde{\mathrm{t}}_{1} \tilde{\mathrm{t}}_{1}$ pair cross section is evaluated to be less than $1 \%$, and the value of $1 \%$ is taken as the systematic error. 
The systematic errors are considered to be independent, and the total systematic error is calculated as a quadratic sum of the individual errors, and was found to be $8 \%-15 \%$, depending on $m_{\tilde{\mathrm{t}}_{1}}$ and $m_{\tilde{\chi}_{1}^{0}}$. In calculating the mass limits, the detection efficiency is reduced by the total systematic error.

\subsection{Mass Limits}

The number of $\tilde{t}_{1} \overline{\tilde{t}}_{1}$ events that would pass our analysis cuts has been calculated as a function of $m_{\tilde{\mathrm{t}}_{1}}, m_{\tilde{\chi}_{1}^{0}}$, and $\theta_{\text {mix }}$. In the data no events survived the cuts, and the region where more than 3.0 events are expected is excluded at $95 \%$ C.L. Figures $3 \mathrm{a}$ and $3 \mathrm{~b}$ show the excluded regions in the $\left(\theta_{\text {mix }}, m_{\tilde{\mathrm{t}}_{1}}\right)$ plane for the mass difference $\Delta m\left(\equiv m_{\tilde{\mathrm{t}}_{1}}-m_{\tilde{\chi}_{1}^{0}}\right) \geq 2 \mathrm{GeV}$, and $\geq 5 \mathrm{GeV}$, respectively, together with the regions excluded by lower energy experiments $[8,9]$.

The decay $Z^{0} \rightarrow \tilde{\mathrm{t}}_{1} \overline{\tilde{\mathrm{t}}}_{1}$, if kinematically allowed, would contribute to the $Z^{0}$ total decay width $\Gamma_{\mathrm{Z}}$ [1]. The limit of the excess of $\Gamma_{\mathrm{Z}}$ with $95 \%$ C.L. above the Standard Model prediction has been calculated to be $\Delta \Gamma_{\mathrm{Z}}<26 \mathrm{MeV}$ from the measured $\Gamma_{Z}$ and its experimental error at LEP [3], taking into account the possible deviations in the theoretical prediction due to the uncertainties in the top quark mass, the Higgs boson mass and $\alpha_{s}$. The region excluded by the limit on the excess of the $\mathrm{Z}^{0}$ total decay width is also shown in the figures $3 \mathrm{a}$ and $3 \mathrm{~b}$.

The numerical mass bounds are listed in Table 3 for three $\theta_{\text {mix }}$ values. Assuming that $\Delta m$ is greater than $2.0 \mathrm{GeV}$, if the $\tilde{\mathrm{t}}_{1}$ is purely left-handed $\left(\theta_{\text {mix }}=0\right)$, the lower mass limit is $41.2 \mathrm{GeV}$ at $95 \%$ C.L. For $\theta_{\text {mix }}=\frac{1}{4} \pi$, the mass region from 6.1 GeV to $38.5 \mathrm{GeV}$, is excluded. For the case where $\tilde{\mathrm{t}}_{1}$ completely decouples to $\mathrm{Z}^{0}\left(\theta_{\mathrm{mix}}=0.98\right)$, a mass region around $15 \mathrm{GeV}$ is excluded.

\begin{tabular}{|r|r|r|r|}
\hline \multicolumn{4}{|c|}{ excluded $m_{\tilde{\mathrm{t}}_{1}}$ region $(\mathrm{GeV})$} \\
\hline$\theta_{\text {mix }}(\mathrm{rad})$ & $\Delta m \geq 2 \mathrm{GeV}$ & $\Delta m \geq 3 \mathrm{GeV}$ & $\Delta m \geq 5 \mathrm{GeV}$ \\
\hline 0.0 & $0.0 \sim 41.2$ & $0.0 \sim 44.9$ & $0.0 \sim 46.0$ \\
\hline$\frac{1}{4} \pi$ & $6.1 \sim 38.5$ & $6.0 \sim 41.8$ & $6.0 \sim 45.2$ \\
\hline 0.98 & $11.2 \sim 25.5$ & $9.5 \sim 31.5$ & $7.9 \sim 41.2$ \\
\hline
\end{tabular}

Table 3: The excluded $m_{\tilde{\mathrm{t}}_{1}}$ region $\left(\Delta m=m_{\tilde{\mathrm{t}}_{1}}-m_{\tilde{\chi}_{1}^{0}}\right)$ with $95 \%$ C.L.

The exclusion zones in the $m_{\tilde{\mathrm{t}}_{1}}-m_{\tilde{\chi}_{1}^{0}}$ plane are shown in Figure 4 for various $\theta_{\text {mix }}$ values. In this figure, it is shown that, for $\Delta m \geq 5 \mathrm{GeV}$ and the mixing angle being smaller than 0.85 rad or greater than $1.15 \mathrm{rad}$, the mass region from 6.1 to $45.1 \mathrm{GeV}$ is excluded at 95\% C.L. As can be seen from these figures, the OPAL exclusion region contains all of the exclusion regions determined from lower energy experiments, except for a small region at low $m_{\tilde{t}_{1}}$. In this region the acoplanarity angle of the two jets is small due to the low momentum of the $\tilde{\chi}_{1}^{0}$ transverse to the $\tilde{t}_{1}$ flight direction and due to large momentum carried by the fragmentation products in the $\tilde{t}_{1}$ flight direction. 


\section{Summary}

Pair production of a supersymmetric partner of the top quark has been searched for using the OPAL detector at the CERN $\mathrm{e}^{+} \mathrm{e}^{-}$collider LEP. No evidence for such events has been found in the data sample taken in 1991-1993 with integrated luminosity of $69.1 \mathrm{pb}^{-1}$, which corresponds to $1.68 \times 10^{6}$ produced $\mathrm{Z}^{0} \rightarrow \mathrm{q} \overline{\mathrm{q}}$ events. The mass limits were obtained with the conservative assumption that only one of the two mass eigenstates of scalar top quarks is observable at LEP. The $\tilde{t}_{1}$ mass is excluded below $45.1 \mathrm{GeV}$ at $95 \%$ C.L., where the mixing angle of left and right-handed partners is smaller than 0.85 rad or greater than $1.15 \mathrm{rad}$, and the mass difference between $\tilde{t}_{1}$ and the lightest neutralino is greater than 5 $\mathrm{GeV}$.

\section{Acknowledgements:}

Sincere thanks are due to K. Hagiwara (KEK) and T. Kon (Seikei Univ.) for useful discussions. It is a pleasure to thank the SL Division for the efficient operation of the LEP accelerator, the precise information on the absolute energy, and their continuing close cooperation with our experimental group. In addition to the support staff at our own institutions we are pleased to acknowledge the Department of Energy, USA, National Science Foundation, USA, Texas National Research Laboratory Commission, USA, Particle Physics and Astronomy Research Council, UK, Natural Sciences and Engineering Research Council, Canada, Fussefeld Foundation, Israeli Ministry of Energy and Ministry of Science, Minerva Gesellschaft,

Japanese Ministry of Education, Science and Culture (the Monbusho) and a grant under the Monbusho International Science Research Program, German Israeli Bi-national Science Foundation (GIF), Direction des Sciences de la Matière du Commissariat à l'Energie Atomique, France,

Bundesministerium für Forschung und Technologie, Germany, National Research Council of Canada, A.P. Sloan Foundation and Junta Nacional de Investigação Científica e Tecnológica, Portugal.

\section{References}

[1] M. Drees and K. Hikasa, Phys. Lett. B252 (1990) 127;

K. Hikasa and M. Kobayashi, Phys. Rev. D36 (1987) 724. 
[2] J. Ellis and S. Rudaz, Phys. Lett. B128 (1983) 248;

G. Altarelli and R. Rückl, Phys. Lett. B144 (1984) 126;

S. Dawson, E. Eichten and C. Quigg, Phys. Rev. D31 (1985) 1581;

J. Ellis, G.L. Fogli and E. Lisi, Nucl. Phys. B393 (1993) 3.

[3] The LEP Collaborations ALEPH, DELPHI, L3 and OPAL, CERN-PPE/93157 (1993).

[4] CDF Collaboration, F. Abe et al., FERMILAB-PUB-94-116-E, May 1994, submitted to Phys. Rev. D.

[5] T. Kon and T. Nonaka, Phys. Lett. B319 (1993) 355.

[6] UA1 Collaboration, C. Albajar et al., Phys. Lett. B198 (1987) 261;

CDF Collaboration, F. Abe et al., Phys. Rev. Lett. 62 (1989) 1825;

UA2 Collaboration, J. Alitti et al., Phys. Lett. B235 (1990) 363;

H.Baer, X. Tata and J. Woodside, Phys. Rev. D44 (1991) 207.

[7] DELPHI Collaboration, P. Abreu et al., Phys. Lett. B247 (1990) 148;

MARK II Collaboration, T. Barklow et al., Phys. Rev. Lett. 64 (1990) 2984.

[8] CELLO Collaboration, H.J. Behrend et al., Zeit. Phys. C35 (1987) 181.

[9] VENUS Collaboration, J. Shirai et al., Phys. Rev. Lett. 72 (1994) 3313.

[10] TOPAZ Collaboration, I. Adachi et al., Phys. Lett. B218 (1989) 105;

AMY Collaboration, Y. Sakai et al., Phys. Lett. B234 (1990) 534.

[11] ALEPH Collaboration, D.Decamp et al., Phys. Lett. B236 (1990) 86;

ALEPH Collaboration, D.Decamp et al., Phys. Report, 216 (1992) 253;

DELPHI Collaboration, P. Aberu et al., Phys. Lett. B247 (1990) 157;

L3 Collaboration, B. Adeva et al., Phys. Lett. B233 (1989) 530;

OPAL Collaboration, M.Z. Akrawy et al., Phys. Lett. B240 (1990) 261.

[12] K. Hagiwara, K. Kato, A.D. Martin, and C.K. Ng, Nucl. Phys. B344 (1990) 1.

[13] I.I. Bigi, V.S. Fadin, and V. Khoze, Nucl. Phys. B377 (1992) 461;

J. Kaplan and J.H. Kühn, Phys. Lett. B78 (1978) 252.

[14] T. Sjöstrand, PYTHIA 5.6 and JETSET 7.3 Physics and Manual, CERN-

TH. 6488/92 (1992);

T. Sjöstrand, Comp. Phys. Comm. 39 (1986) 347;

T. Sjöstrand and M. Bengtsson, Comp. Phys. Comm. 43 (1987) 367.

[15] B. Andersson et al., Phys. Rep. 97 (1983) 31. 
[16] OPAL Collaboration, M.Z. Akrawy et al., Zeit. Phys. C47 (1990) 505.

[17] C. Peterson, D. Schlatter, I. Schmitt and P.M. Zerwas, Phys. Rev. D27 (1983) 105.

[18] A. Ali, Zeit. Phys. C1 (1979) 25;

M.K. Gaillard, B.W. Lee and J.L. Rosner, Rev. Mod. Phys. 47 (1975) 227;

V. Barger, T. Gottschalk and R.J.N Phillips, Phys. Lett. B82 (1979) 445;

M. Suzuki, Nucl. Phys. B258 (1985) 553.

[19] OPAL Collaboration, K. Ahmet et al., Nucl. Inst. Methods A305(1991) 275.

[20] OPAL Collaboration, G. Alexander et al., Zeit. Phys. C52(1991) 175.

[21] S. Jadach et al., Z Physics at LEP 1, CERN 89-08, ed. G. Altarelli et al., Vol 1 (1989) 235.

[22] A. Buijs, W.G.J. Langeveld, M.H. Letho and D.J. Miller, CERN-PPE/93-199, submitted to Comp. Phys. Comm.

[23] M.G. Bowler, Zeit. Phys. C11 (1981) 169.

[24] OPAL Collaboration, P. D. Acton et al., Zeit. Phys. C58 (1993) 523. 
Figure 1: The plots for the light $\tilde{\mathrm{t}}_{1}$ search for the data, typical simulated $\tilde{\mathrm{t}}_{1} \overline{\tilde{t}}_{1}$ events $\left(m_{\tilde{\mathrm{t}}_{1}}=20 \mathrm{GeV}, m_{\tilde{\chi}_{1}^{0}}=10 \mathrm{GeV}\right)$, and the simulated background events (events due to hadronic $\mathrm{Z}^{0}$ decays, $\mathrm{Z}^{0}$ decays to $\tau$ pairs, and two photon collisions). The corresponding cross section of the $\tilde{t}_{1} \overline{\tilde{t}}_{1}$ events in these figures is about $30 \mathrm{pb}$ assuming the luminosity of $69.1 \mathrm{pb}^{-1}$.

(a) The scatter plot of the visible energy $E_{\text {vis }}$ and the acoplanarity angle $\theta_{\text {Acop }}$ before the $E_{\text {vis }}$ cut. The events between the two vertical lines are selected by the $E_{\text {vis }}$ cut.

(b) The scatter plot of the jet-direction $\left|\cos \theta_{\text {jet }}\right|$ and $\theta_{\text {Acop }}$ after the $E_{\text {vis }}$ cut. The line shows the final cuts on $\theta_{\text {Acop }}$ and $\left|\cos \theta_{\text {jet }}\right|$.

Figure 2: The plots for the heavy $\tilde{\mathrm{t}}_{1}$ search for the data, typical simulated $\tilde{\mathrm{t}}_{1} \overline{\tilde{t}}_{1}$ events $\left(m_{\tilde{\mathrm{t}}_{1}}=42 \mathrm{GeV}, m_{\tilde{\chi}_{1}^{0}}=32 \mathrm{GeV}\right)$, and the simulated background events (events due to hadronic $\mathrm{Z}^{0}$ decays, $\mathrm{Z}^{0}$ decays to $\tau$ pairs, and two photon collisions). The corresponding cross section of the $\tilde{t}_{1} \tilde{\tilde{t}}_{1}$ events in these figures is about $30 \mathrm{pb}$ assuming the luminosity of $69.1 \mathrm{pb}^{-1}$.

(a) The scatter plot of the visible energy $E_{\text {vis }}$ and the acoplanarity angle $\theta_{\text {Acop }}$ before the $E_{\text {vis }}$ cut. The events between the two vertical lines are selected by the $E_{\text {vis }}$ cut.

(b) The scatter plot of the jet-direction $\left|\cos \theta_{\text {jet }}\right|$ and $\theta_{\text {Acop }}$ after the $E_{\text {vis }}$ cut. The line shows the final cuts on $\theta_{\text {Acop }}$ and $\left|\cos \theta_{\text {jet }}\right|$.

Figure 3: The excluded region in the $\left(\theta_{\text {mix }}, m_{\tilde{\mathrm{t}}_{1}}\right)$ plane at $95 \%$ C.L. where the mass difference is assumed to be:

(a) $m_{\tilde{\mathrm{t}}_{1}}-m_{\tilde{\chi}_{1}^{0}} \geq 2 \mathrm{GeV}$,

(b) $m_{\tilde{\mathrm{t}}_{1}}-m_{\tilde{\chi}_{1}^{0}} \geq 5 \mathrm{GeV}$.

The region excluded from the limit on the $\mathrm{Z}^{0}$ total decay width $\left(\Delta \Gamma_{\mathrm{Z}} \leq 26 \mathrm{MeV}\right.$ at $95 \%$ C.L.) and limits from previous publications $[8,9]$ are also shown.

Figure 4: The excluded region in the $\left(m_{\tilde{\mathrm{t}}_{1}}, m_{\tilde{\chi}_{1}^{0}}\right)$ plane at $95 \%$ C.L. where the mixing angle is assumed to be $\theta_{\text {mix }} \leq 0.85$ or $\geq 1.15$ rad (shaded area), and $\theta_{\text {mix }} \leq 0.97$ or $\geq 0.99$ rad (hatched area).

The dashed curve shows the contour of the limits from previous publications $[8,9]$. 